\title{
Tomographic reconstruction of atmospheric gravity wave parameters from airglow observations
}

\author{
Rui Song ${ }^{1,2}$, Martin Kaufmann ${ }^{1}$, Jörn Ungermann ${ }^{1}$, Manfred Ern ${ }^{1}$, Guang Liu ${ }^{3}$, and Martin Riese ${ }^{1,2}$ \\ ${ }^{1}$ Institute of Energy and Climate Research, Stratosphere (IEK-7), Research Centre Jülich, 52425 Jülich, Germany \\ ${ }^{2}$ Institute for Atmospheric and Environmental Research, University of Wuppertal, 42119 Wuppertal, Germany \\ ${ }^{3}$ Key Laboratory of Digital Earth Sciences, Institute of Remote Sensing and Digital Earth, \\ Chinese Academy of Sciences, Beijing, China
}

Correspondence to: Rui Song (r.song@fz-juelich.de)

Received: 18 April 2017 - Discussion started: 21 April 2017

Revised: 27 August 2017 - Accepted: 21 October 2017 - Published: 30 November 2017

\begin{abstract}
Gravity waves (GWs) play an important role in the dynamics of the mesosphere and lower thermosphere (MLT). Therefore, global observations of GWs in the MLT region are of particular interest. The small scales of GWs, however, pose a major problem for the observation of GWs from space. We propose a new observation strategy for GWs in the mesopause region by combining limb and sub-limb satellite-borne remote sensing measurements for improving the spatial resolution of temperatures that are retrieved from atmospheric soundings. In our study, we simulate satellite observations of the rotational structure of the $\mathrm{O}_{2}$ A-band nightglow. A key element of the new method is the ability of the instrument or the satellite to operate in so-called "target mode", i.e. to point at a particular point in the atmosphere and collect radiances at different viewing angles. These multi-angle measurements of a selected region allow for tomographic 2-D reconstruction of the atmospheric state, in particular of GW structures. The feasibility of this tomographic retrieval approach is assessed using simulated measurements. It shows that one major advantage of this observation strategy is that GWs can be observed on a much smaller scale than conventional observations. We derive a GW sensitivity function, and it is shown that "target mode" observations are able to capture GWs with horizontal wavelengths as short as $\sim 50 \mathrm{~km}$ for a large range of vertical wavelengths. This is far better than the horizontal wavelength limit of 100$200 \mathrm{~km}$ obtained from conventional limb sounding.
\end{abstract}

\section{Introduction}

Miniaturization in remote sensing instrumentation as well as spacecraft technology allows for the implementation of highly focused satellite missions, for example to observe airglow layers in the mesosphere and lower thermosphere (MLT) region. The MLT extends between about 50 and $110 \mathrm{~km}$ in the Earth's atmosphere, and is highly affected by atmospheric waves, including planetary waves, tides, and gravity waves (GWs), which are mainly excited in the lower atmosphere (Vincent, 2015). Atmospheric GWs are the main driver for the large-scale circulation in the MLT region with considerable effects on the atmospheric state and temperature structure (Garcia and Solomon, 1985; Holton, 1982; Lindzen, 1981).

Temperature is a key quantity to describe the atmospheric state, and it is a valuable indicator to identify and quantify atmospheric waves, such as GWs (e.g. Fritts and Alexander, 2003, and reference therein). As GWs displace air parcels adiabatically both in vertical and horizontal directions, this process affects the temperature of the atmosphere. Assuming linear wave theory, GW-related fluctuations in different parameters (wind, temperature, density, etc.) are directly connected via the linear polarization relations (e.g. Fritts and Alexander, 2003). Therefore, amplitudes, wavelengths, and phases of a GW can be determined from its temperature structure (Fritts et al., 2014; Ern et al., 2004, 2017).

Over the last few decades, data from satellite and aircraft instruments have been extensively used to characterize vertically resolved GW parameters. Utilizing limb soundings, these data sets include temperature or density data acquired 
by the Limb Infrared Monitor of the Stratosphere (LIMS) (Fetzer and Gille, 1994, 1996), the Global Positioning System (GPS) radio occultation (RO) (Tsuda et al., 2000), Cryogenic Infrared Spectrometers and Telescopes for the Atmosphere (CRISTA) (Eckermann and Preusse, 1999), Sounding of the Atmosphere using Broadband Emission Radiometry (SABER) (Ern et al., 2011; Preusse et al., 2009a), High Resolution Dynamics Limb Sounder (HIRDLS) (Alexander et al., 2008), and Gimballed Limb Observer for Radiance Imaging of the Atmosphere (GLORIA) aircraft (Riese et al., 2014; Kaufmann et al., 2015; Ungermann et al., 2010b, 2011). GWs can be also characterized by nadir-viewing instruments, such as the Atmospheric Infrared Sounder (AIRS) (Alexander and Barnet, 2007; Hoffmann and Alexander, 2009; Gong et al., 2012; Hoffmann et al., 2016; Ern et al., 2017), and the Advanced Microwave Sounding Unit (AMSU) (Wu, 2004).

Typical limb sounders provide middle atmosphere temperature data with a vertical resolution of $1-3 \mathrm{~km}$ assuming a horizontally homogeneous atmosphere. In most cases, vertical structures of small horizontal-scale GWs are characterized by separating a background temperature profile from the measured profiles. The average temperature structure of the atmosphere, the tides (e.g. Forbes et al., 2006) and several different modes of planetary waves (e.g. Ern et al., 2009) contribute to this background temperature. The final results of this procedure are altitude profiles of temperature perturbations due to GWs. Temperature data obtained from limb sounding instruments exhibit a very good vertical resolution, but suffer from a poor horizontal resolution along the instrument's line of sight, thus limiting the visibility of waves with short horizontal wavelengths. Ern et al. (2004) proposed to combine the phases provided by the wave analysis of adjacent temperature vertical profiles to estimate the horizontal wavelength of GWs. This approach has been successfully applied to retrieve GWs with vertical wavelengths between 6 and $30 \mathrm{~km}$ and horizontal wavelengths larger than $100 \mathrm{~km}$ from CRISTA-2 measurements. The method has also been used for several other data sets (Alexander et al., 2008; Wright et al., 2010; Ern et al., 2011).

A general limitation of all methods based on limb sounding is the poor along-line-of-sight resolution of this kind of measurement, which is typically a few hundred kilometres. A few existing and upcoming limb sounders try to mitigate this general limitation by considering the horizontal variability of the atmosphere in the retrieval (Livesey and Read, 2000; Carlotti et al., 2001; von Clarmann et al., 2009). The GLORIA limb sounder utilizes a tomographic reconstruction technique, which leads to a horizontal resolution of $20 \mathrm{~km}$ (Ungermann et al., 2010b). In this work, we present another measurement strategy to detect atmospheric small structures, whose spatial dimensions are neither covered by conventional limb sounding nor satellite- or ground-based nadir sounding. It is applicable to a low-cost nanosatellite utilizing a remote sensing instrument to measure atmospheric temperature and a high-precision pointing system. Simply speaking, the satellite is commanded in such a way that the instrument observes a certain volume in the atmosphere multiple times while the satellite is flying by. This results in multi-angle observations of the target volume, such that a tailored retrieval scheme can be applied. This differs from classical limitedangle tomography, where only observations within a limited angular range are taken for the reconstruction.

In Sect. 2, we present the observation strategy which we call "target mode" observations. Section 3 describes the forward modelling of such "target mode" measurements, which is based on a 2-D ray tracing, an oxygen atmospheric band (A-band) airglow emission model, a GW perturbation, and the corresponding radiative transfer. The retrieval algorithm is presented in Sect. 4. In Sect. 5, the performance of the "target mode" tomographic retrieval is tested with simulated measurements. A sensitivity study is used to analyse its performance in deriving GW fine structures compared with pure limb tomographic retrieval. The conclusion is given in Sect. 6.

\section{Observation strategy}

The detection of small-scale structures in Earth's atmosphere in 2-D requires new instruments or measurement strategies, as stated above. One of those is to observe atmospheric volumes from different viewing directions - e.g. by pointing at one particular region while the instrument is flying by. Such tomographic retrievals have been demonstrated and implemented in a variety of measurements for different purposes, including by Carlotti et al. (2001) for Michelson Interferometer for Passive Atmospheric Sounding (MIPAS), Livesey and Read (2000) for MLS, Ungermann et al. (2010a) for Process Exploration through Measurements of Infrared and millimetre-wave Emitted Radiation (PREMIER), and Ungermann et al. (2011) and Kaufmann et al. (2015) for the airborne GLORIA instrument. In this work, we propose to combine satellite-borne limb and sub-limb measurements of a nightglow layer in such a way that we obtain multi-angle observations of a particular air volume as well. The sub-limb sounding has a similar geometry to limb sounding, whereas the tangent heights are near or below the surface. We name this combined observation strategy "target mode", i.e. we adopt the same expression as for similar measurements in Earth observations. In the following, the capabilities of "target mode" observations will be discussed for a specific sequence of satellite pointing manoeuvres.

Figure 1 illustrates the viewing geometry of the "target mode" observation, which incorporates limb and sub-limb sounding measurements. When the satellite is operated in "target mode", the instrument will start to observe the target atmospheric volume by forward limb imaging first. The instrument will continue to measure under limb geometry for a period of time, and multiple consecutive vertical radiance profiles will be taken during this time. Then, the instrument 


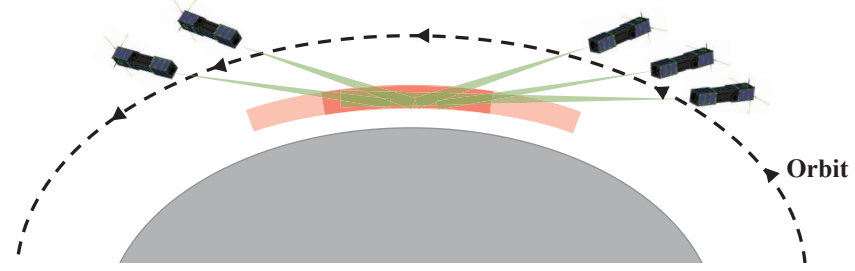

Figure 1. Viewing geometry of "target mode" observations of a region in a mesospheric emission layer. This observation mode consists of forward limb, forward sub-limb and backward sub-limb measurements. The sub-limb measurements are taken with two different viewing angles $-24.5^{\circ}$ when the satellite is far from the target region and $33^{\circ}$ when it is closer. The viewing angle is the angle relative to the instantaneous horizon, with horizon defined as Earth surface for a given but temporally changing satellite position.

will switch to a forward sub-limb view with a $24.5^{\circ}$ viewing angle below horizon. This viewing angle is also constant during the sub-limb observations. In this way, the volume will be scanned twice by the limb and sub-limb observations. Depending on the flexibility and possible speed of satellite operations, more viewing angle positions could be used - for example another position with a viewing angle $>24.5^{\circ}$ as indicated in Fig. 1. After the satellite overpasses the target volume, the same measurement sequence will be applied by back-looking at the target volume.

Figure 2 shows how the lines of sight (LOSs) of the measurements overlap with each other in the orbit plane under limb sounding and "target mode", respectively. For an assumed orbit altitude of $600 \mathrm{~km}$, the corresponding measurement time for taking the measurement sequences for the limb sounding mode shown in Fig. 2a is $1.6 \mathrm{~min}$. We further assume a high measurement frequency of $10 \mathrm{~s}$ per vertical profile. As can be seen in Fig. 2a, for the limb sounding mode the LOSs of consecutive limb profiles overlap, which means that the same atmospheric volume is observed from different directions. In the "target mode" (Fig. 2b), sub-limb measurements contribute further information by intersecting the observed volume at different viewing angles about $3.3 \mathrm{~min}$ after the limb observations were taken.

\section{Forward model}

\section{$3.1 \quad \mathrm{O}_{2}$ A-band nightglow emission model}

The observation strategy presented in the previous section requires that the observed emission be restricted to a limited altitude range and that any emissions from lower parts of the atmosphere or Earth's surface cannot reach the instrument. This requires that the atmosphere below the emission layer be optically thick for those emissions. This limits the number of potential airglow emissions significantly, because most of them are hotband transitions between two excited vibrational states. The number density of the lower state of a hotband transition is typically too low to absorb background radiation from the lower atmosphere. Therefore, we have to search for airglow emissions, whose lower state is a ground state of a frequent atmospheric species. This is the case for the $\mathrm{O}_{2}$ A-band nightglow emission. The emitting electronic state is excited in a two-step Barth process (Burrage et al., 1994; Greer et al., 1981):

$\mathrm{O}+\mathrm{O}+M \rightarrow \mathrm{O}_{2}^{*}+M$,

where $M$ is an $\mathrm{O}_{2}$ or $\mathrm{N}_{2}$ molecule, and $\mathrm{O}_{2}^{*}$ is an excited $\mathrm{O}_{2}$ molecule. Then, the $\mathrm{O}_{2}^{*}$ state is quenched to a lower electronic state $\mathrm{O}_{2}\left(b^{1} \Sigma\right)$, which emits $\mathrm{O}_{2} \mathrm{~A}$-band radiation:

$\mathrm{O}_{2}^{*}+\mathrm{O}_{2} \rightarrow \mathrm{O}_{2}\left(b^{1} \Sigma\right)+\mathrm{O}_{2}$.

Loss mechanisms for both $\mathrm{O}_{2}\left(b^{1} \Sigma\right)$ and its undefined precursor $\mathrm{O}_{2}^{*}$ include quenching by $\mathrm{O}_{2}, \mathrm{O}_{3}, \mathrm{~N}_{2}$, or $\mathrm{O}$ and spontaneous emission. The A-band volume emission rate (VER) $\eta$, in photons $\mathrm{s}^{-1} \mathrm{~cm}^{-3}$, is thus (McDade et al., 1986)

$$
\eta=\frac{A_{1} k_{1}[\mathrm{O}]^{2}[M]\left[\mathrm{O}_{2}\right]}{\left(A_{2}+k_{2}\left[\mathrm{O}_{2}\right]+k_{3}\left[\mathrm{~N}_{2}\right]+k_{4}[\mathrm{O}]\right)\left(\mathrm{C}_{\mathrm{O}_{2}}\left[\mathrm{O}_{2}\right]+\mathrm{Co}_{\mathrm{O}}[\mathrm{O}]\right)},
$$

where [] refers to the number density of the species within the brackets. $A_{1}$ is the A-band transition probability, $A_{2}$ is the total transition probability of the zeroth vibrational level of the $\mathrm{O}_{2}\left(b^{1} \Sigma\right)$ state (Vallance Jones, 1974). $k_{1}$ is the reaction coefficient rate for reaction Eq. (1) (Campbell and Gray, 1973). The quenching rates for $\mathrm{O}_{2}, \mathrm{~N}_{2}$, and $\mathrm{O}$ are denoted by $k_{2}, k_{3}$, and $k_{4}$, respectively. $\mathrm{C}_{\mathrm{O}_{2}}$ and $\mathrm{C}_{\mathrm{O}}$ describe quenching rates of $\mathrm{O}_{2}^{*}$ by $\mathrm{O}_{2}$ and $\mathrm{O}$. All rate constants utilized in this work are taken from Sheese (2011).

A typical vertical profile of a modelled $\mathrm{O}_{2}$ A-band nightglow emission from 80 to $110 \mathrm{~km}$ is shown in Fig. 3 . The temperature $T$ and number densities of $\mathrm{O}_{2}, \mathrm{~N}_{2}$, and $\mathrm{O}$ are taken from the Hamburg Model of the Neutral and Ionized Atmosphere (HAMMONIA) (Schmidt et al., 2006) model run at $30^{\circ} \mathrm{N}$ and $88^{\circ} \mathrm{E}$ for $22: 00$ local solar time. The intensity of the $\mathrm{O}_{2}$ A-band nightglow limb emission peaks at around $93 \mathrm{~km}$; typical peak values are $3 \times 10^{3}$ photons s $\mathrm{s}^{-1} \mathrm{~cm}^{-3}$.

Since the lifetime of the $\mathrm{O}_{2}\left(b^{1} \Sigma\right)$ state is more than $12 \mathrm{~s}$, it can be assumed that the molecule is in rotational localthermodynamic equilibrium (Vallance Jones, 1974). This allows us to derive the kinetic temperature of the atmosphere from the rotational band structure of the emissions. Under thermal equilibrium conditions, the $\mathrm{O}_{2} \mathrm{~A}$-band rotational excitation follows the Boltzmann distribution at a rotational temperature $T$, which is assumed to be equal to the background temperature. The number of photons that appears in an individual rotational line is given by $\eta_{\text {rot }}$

$\eta_{\text {rot }}=\eta \frac{g^{\prime}}{Q(T)} \exp \left(\frac{-h c E^{\prime}}{k T}\right) A_{1}$,

where $h$ is the Planck constant, $c$ is the light speed, and $k$ is the Boltzmann constant. $E^{\prime}$ and $g^{\prime}$ are the upper state energy 

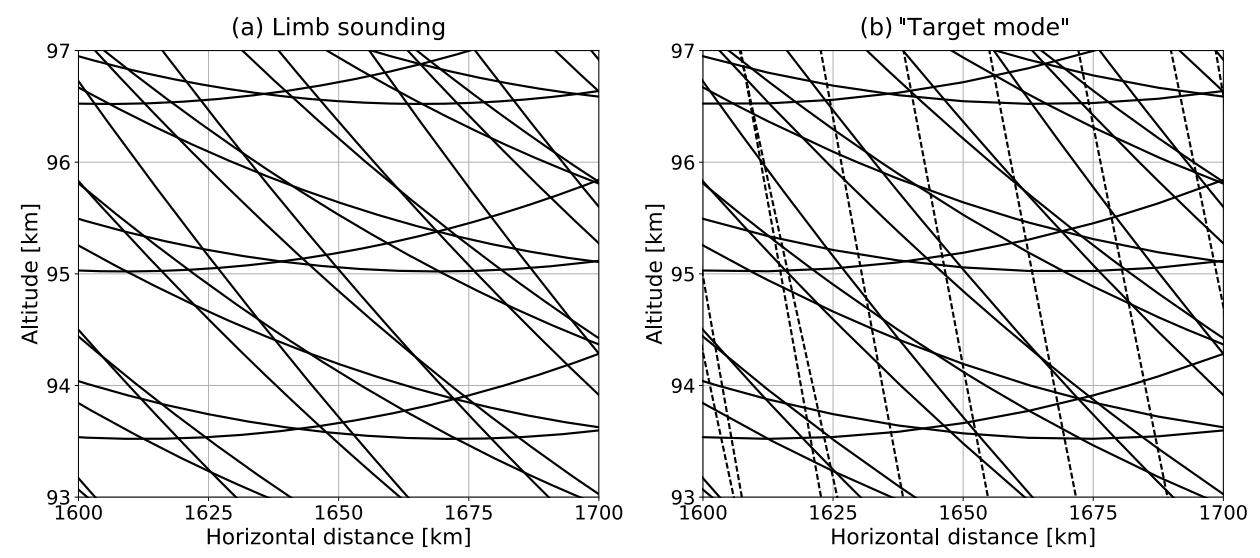

Figure 2. Central measurement track of an imaging instrument in the pure limb sounding and "target mode". The solid lines represent limb measurements and dashed lines represent sub-limb measurements. The satellite viewing geometry of (b) is the same as in Fig. 1.

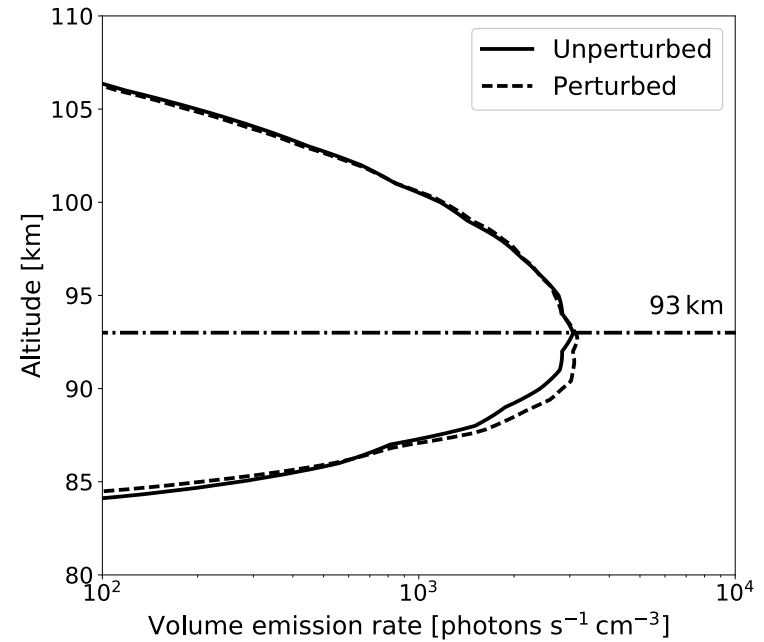

Figure 3. Modelled $\mathrm{O}_{2}$ A-band nightglow emission profile at $30^{\circ} \mathrm{N}$ and $88^{\circ}$ E for 22:00 local solar time as simulated by the HAMMONIA model. The solid curve represents an $\mathrm{O}_{2}$ A-band profile for unperturbed conditions, and the dashed curve represents a profile perturbed by a GW with $15 \mathrm{~km}$ vertical wavelength and $5 \mathrm{~K}$ amplitude.

and upper state degeneracy, respectively. $A_{1}$ is the Einstein coefficient of the transition. $Q(T)$ is the rotational partition function:

$Q(T)=\sum g^{\prime} \exp \left(\frac{-h c E^{\prime}}{k T}\right)$

A subset of six emission lines has proven to give an optimal setup for a potential satellite mission aiming to the derivation of kinetic temperature from the $\mathrm{O}_{2} \mathrm{~A}$ band. These six lines show both positive and negative temperature dependence of rotational structures; also strong, medium and weak dependence are included, as shown in Fig. 4.

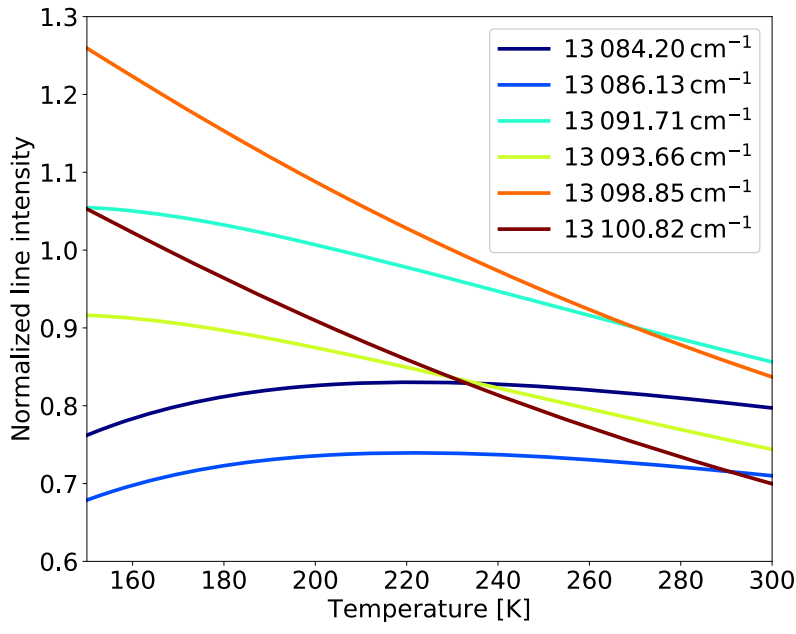

Figure 4. Temperature dependence of six rotational lines of the $\mathrm{O}_{2}$ A band. The line centre wavenumbers for the lines are given in the figure legend. The intensity is normalized around the maximum intensity for a temperature of $230 \mathrm{~K}$.

\subsection{Wave perturbations}

$\mathrm{O}_{2}$ A-band emissions are affected by GWs due to the vertical displacement of constituents and temperature changes associated with the waves. Following conventional assumption, we consider an adiabatic and windless atmosphere. A monochromatic wave perturbation added in background temperature $T_{0}$ at position $(x, z)$ can be written as

$T(x, z, t)=T_{0}(x, z, t)+A \cos \left(\frac{2 \pi x}{\lambda_{x}}+\frac{2 \pi z}{\lambda_{z}}-\hat{\omega} t\right)$,

with wave amplitude $A$, vertical wavelength $\lambda_{z}$, horizontal wavelength $\lambda_{x}$, and $\hat{\omega}$ the intrinsic frequency of wave perturbation. We used the following expression (Ward, 1999) to calculate the vertical displacement $\delta z$ of an air parcel from 


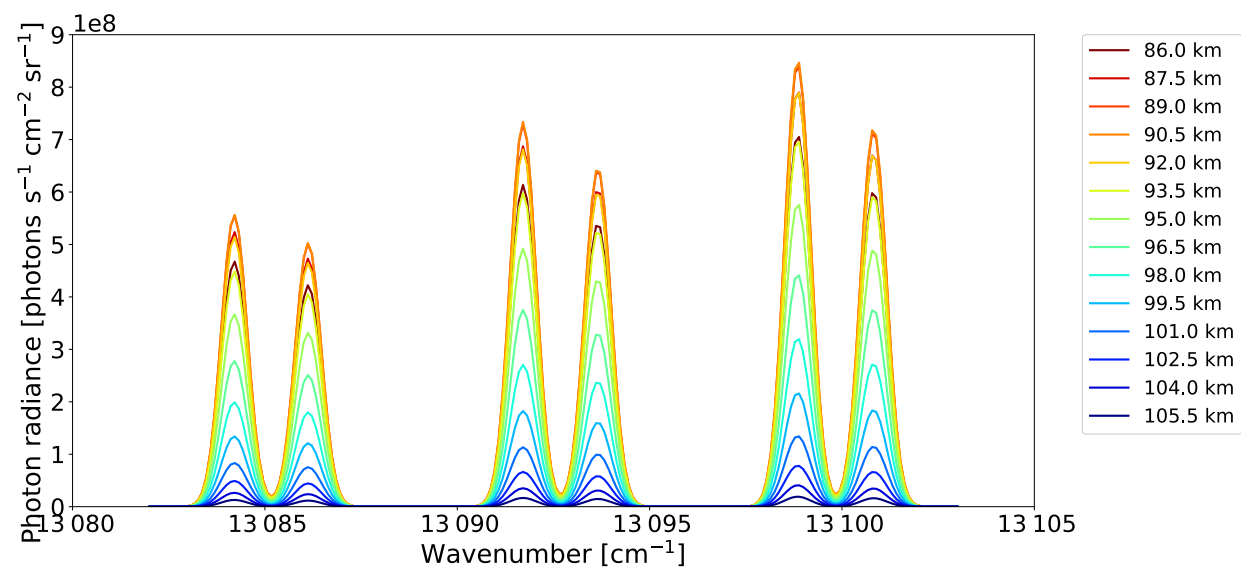

Figure 5. Modelled limb spectral photon radiance for different tangent altitudes. The background atmosphere is taken from the HAMMONIA model, around $30^{\circ} \mathrm{N}$ and $88^{\circ} \mathrm{E}$ for $22: 00$ local solar time.

its equilibrium height $z+\delta z$ :

$T(x, z, \delta z) \approx T(x, z)+\left(\Gamma_{\mathrm{ad}}-\Gamma\right) \delta z$,

where $\Gamma$ and $\Gamma_{\text {ad }}$ are the local and adiabatic lapse rates, respectively. Then, the perturbed density (background density plus perturbation) $\rho^{\prime}$ at fixed height $z$ can be calculated as density at equilibrium height $z+\delta_{z}$ :

$\rho^{\prime}(x, z)=\rho(x, z, \delta z) \approx \rho(x, z) \exp ^{-\kappa \delta z / H}$,

with the scale height $H$. In the quantity $\kappa=\left(c_{p} / c_{v}-1\right)$, the $c_{p}$ and $c_{v}$ represent heat capacities at constant pressure and volume, respectively. Given $\rho^{\prime}$, the number densities for perturbed major gases are calculated as (Liu and Swenson, 2003; Vargas et al., 2007)

$\frac{\left[\mathrm{N}_{2}\right]^{\prime}}{\left[\mathrm{N}_{2}\right]}=\frac{\left[\mathrm{O}_{2}\right]^{\prime}}{\left[\mathrm{O}_{2}\right]}=\frac{\rho^{\prime}}{\rho}$.

Because the mixing ratio of atomic oxygen is not constant with altitude, the perturbed volume mixing ratio $v^{\prime}$ is calculated as follows (Ward, 1999):

$v_{\mathrm{O}}^{\prime}(x, z)=v_{\mathrm{O}}\left(x, z, \delta_{z}\right) \approx v_{\mathrm{O}}\left(x, z+\delta_{z}\right)$.

Figure 3 shows a perturbed $\mathrm{O}_{2} \mathrm{~A}$-band volume emission rate profile perturbed by a 1-D GW with a vertical wavelength $\lambda_{z}$ of $15 \mathrm{~km}$ and an amplitude $A$ of $5 \mathrm{~K}$.

\subsection{Ray tracing}

To model the instrument's LOS in a 3-D atmosphere we utilize a module of the Atmospheric Radiative Transfer Simulator (ARTS) (Buehler et al., 2005). ARTS is a free opensource software program that simulates atmospheric radiative transfer. It focuses on thermal radiation from the microwave to the infrared spectral range. The second version of ARTS (Eriksson et al., 2011) allows simulations for 1-D,
2-D or 3-D atmospheres. In this study, the relative orientation of LOS is selected to be parallel to the orbit plane. Thus, it is assumed that the LOS is in the orbit plane, and a 2-D ray tracing can be applied with ARTS-2.

\subsection{Radiative transfer}

The observed spectral irradiance $I(v)$, in photons $\mathrm{s}^{-1} \mathrm{~cm}^{-2}$, is a path integral along the line of sight:

$I(v)=\int_{-\infty}^{\infty} \eta(s)_{\text {rot }} D(v, s) \exp \left[-\int_{-s}^{\infty} n\left(s^{\prime}\right) \sigma\left(s^{\prime}\right) \mathrm{d} s^{\prime}\right] \mathrm{d} s$,

where $s$ is the distance along the line-of-sight, $n$ is the $\mathrm{O}_{2}$ number density, $\sigma$ is the absorption cross-section, and $D(v)$ is the Doppler line shape for the spectral line centred at wavenumber $v$. In the infrared the lower atmosphere is optically thick, whereas the upper atmosphere can be considered as optically thin. Since the $\mathrm{O}_{2} \mathrm{~A}$ band is a transition to the $\mathrm{O}_{2}$ ground state, the atmosphere becomes optically thick at stratopause altitudes. Therefore, any emission from the Earth's surface or tropospheric altitudes cannot reach the upper mesosphere at these wavelengths. At nightglow layer altitudes (upper mesosphere/lower thermosphere) the atmosphere is optically thin for the wavelengths considered. In our case, for altitudes above $85 \mathrm{~km}$, the atmosphere is assumed to be optically thin and the self-absorption term in Eq. (11) can be omitted.

The spectral range considered in this work is 13082 $13103 \mathrm{~cm}^{-1}$ and contains six emission lines. The central wavenumbers of these lines are given in Fig. 4. In Fig. 5, limb spectral photon radiance is simulated at different altitudes (86-115.5 km with $1.5 \mathrm{~km}$ interval). To make a tradeoff between bandwidth and instrument size, a spectral resolution of $0.8 \mathrm{~cm}^{-1}$ is chosen. The temperature dependence of the lines in this spectral interval is illustrated in Fig. 4. 

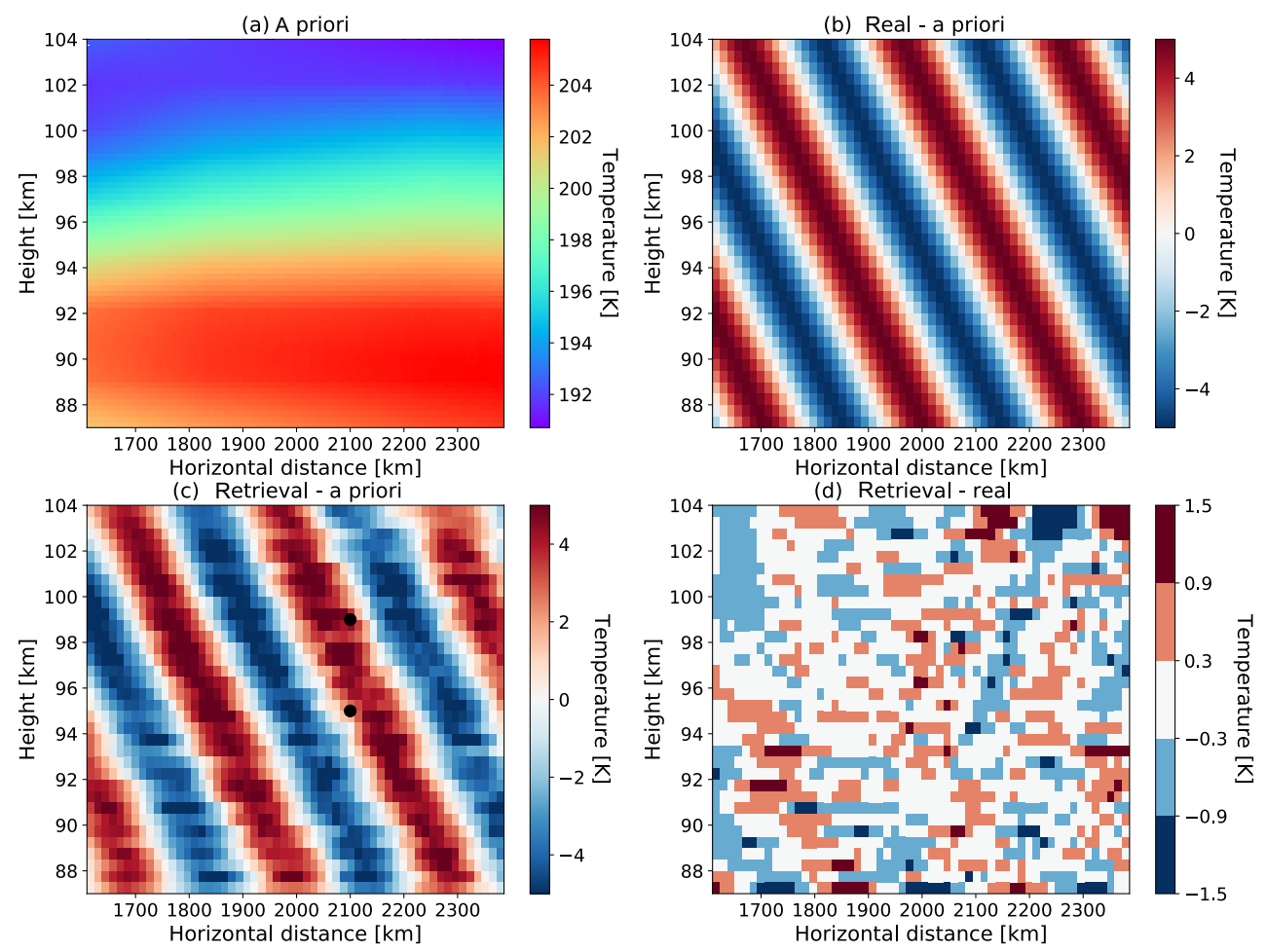

Figure 6. Retrieval result using simulated data. The background atmosphere is taken from the HAMMONIA run between $30^{\circ}$ and $36^{\circ} \mathrm{N}$, $90^{\circ} \mathrm{E}$, around 22:00 local solar time. The a priori atmosphere is depicted in panel (a). The difference between the perturbed atmosphere and the a priori is shown in panel (b). The retrieved wave perturbation, which is obtained by subtracting the a priori from the retrieval result, is given in panel (c). The two black dots correspond to the retrieval points selected for Fig. 7. The difference between the retrieval result and the true state of atmosphere is shown in panel (d).

\section{Retrieval model}

The tomographic retrieval presented here is similar to the widely used optimal estimation approach (Rodgers, 2004). The measurement space is represented by vector $\boldsymbol{y}$ and the unknown atmospheric state is represented by vector $\boldsymbol{x}$. The forward model $\boldsymbol{f}(\boldsymbol{x})$ provides the simulated spectrum based on a given atmospheric state $\boldsymbol{x}$ :

$y=f(x)+\epsilon$,

where $\boldsymbol{\epsilon}$ is the measurement error. The inversion problem of Eq. (12) is generally ill-posed, and the solution is not unique. Following Tikhonov and Arsenin (1977) and Rodgers (2004), the cost function $\boldsymbol{J}$ is complemented by a regularization term:

$$
\begin{aligned}
\boldsymbol{J}(\boldsymbol{x})= & (\boldsymbol{f}(\boldsymbol{x})-\boldsymbol{y})^{T} \mathbf{S}_{\epsilon}^{-1}(\boldsymbol{f}(\boldsymbol{x})-\boldsymbol{y}) \\
& +\left(\boldsymbol{x}-\boldsymbol{x}_{a}\right)^{T} \mathbf{R}\left(\boldsymbol{x}-\boldsymbol{x}_{a}\right),
\end{aligned}
$$

where matrix $\mathbf{R}$ is the inverse covariance or regularization matrix, $\mathbf{S}_{\epsilon}$ is the covariance matrix of the measurement error, and $\boldsymbol{x}_{a}$ represents the a priori data. The a priori data are usually taken as the climatological mean of the retrieved quantities. The second term in the cost function (Eq. 13) ensures that a unique and physically meaningful solution can be obtained. The inversion of the forward model (Eq. 12) can be formulated as a minimization of the cost function $\boldsymbol{J}(\boldsymbol{x})$ given in Eq. (13). To solve the non-linear minimization, we adopt a Levenberg-Marquardt iteration scheme (Levenberg, 1944; Marquardt, 1963; Ceccherini and Ridolfi, 2010).

\subsection{2-D regularization matrix}

The design of the 2-D regularization matrix $\mathbf{R}$ in Eq. (13) is of considerable importance to the retrieval results. Here, we used a combination of zeroth- and first-order Tikhonov regularization (Tikhonov and Arsenin, 1977):

$\mathbf{R}=\left(\alpha_{0} \mathbf{L}_{0}^{T} \mathbf{L}_{0}+\alpha_{1}^{x} \mathbf{L}_{1}^{x T} \mathbf{L}_{1}^{x}+\alpha_{1}^{y} \mathbf{L}_{1}^{y T} \mathbf{L}_{1}^{y}\right)$,

where the weighting parameters $\alpha_{0}, \alpha_{1}^{x}$, and $\alpha_{1}^{y}$ control the overall strength of the regularization term added in Eq. (13). Large values of weighting parameters will result in an overregularized result, while a small value will give an unstable solution. The parameter $\alpha_{0}, \alpha_{1}^{x}$ and $\alpha_{1}^{y}$ also balance the contribution of the zeroth- and the two directional first-order regularization terms. $\mathbf{L}_{0}$ is an identity matrix that constrains the result to the absolute value of $\boldsymbol{x}_{a}$. Matrix $\mathbf{L}_{1}$ maps $\boldsymbol{x}$ onto its 
first-order derivative in the vertical and horizontal directions:

$$
\begin{aligned}
& \mathbf{L}_{1}^{x}(i, j)= \begin{cases}1 & \text { if } j=i+1 \\
-1 & \text { if } j=i \\
0 & \text { otherwise }\end{cases} \\
& \mathbf{L}_{1}^{y}(i, j)= \begin{cases}1 & \text { if } j=i+m \\
-1 & \text { if } j=i \\
0 & \text { otherwise. }\end{cases}
\end{aligned}
$$

As we convert the 2-D atmospheric temperature to a vector $\boldsymbol{x}$ row by row, $\mathbf{L}_{1}^{x}$ is thus a $(l-1) \times l$ matrix, with $l$ the number of elements in $\boldsymbol{x} . \mathbf{L}_{1}^{y}$ is a $(l-m) \times l$ matrix with $m$ to be the number of elements contained in each row of the 2-D atmospheric volume.

\subsection{Averaging kernel matrix}

Following the concept of Rodgers (2004), the effect of the regularization onto the retrieval result can be quantified by the averaging kernel (AVK) matrix:

$\mathbf{A}=\left(\mathbf{R}+\mathbf{K}^{T} \mathbf{S}_{\epsilon}^{-1} \mathbf{K}\right)^{-1} \mathbf{K}^{T} \mathbf{S}_{\epsilon}^{-1} \mathbf{K}$,

where $\mathbf{K}$ is the Jacobian of the forward model $f$ at atmospheric state $\boldsymbol{x}$. The measurement contribution vector can be obtained from the AVK by the sum over each row of $\mathbf{A}$. If the measurement contribution value is close to 1 , most information of the retrieval result is determined by the measurements and not by the absolute value of the a priori data. The averaging kernel matrix can also be used to deduce the spatial resolution of retrieval result. For 1-D retrievals, the vertical resolution is described by calculating the full width at half maximum (FWHM) of the corresponding row of the AVK matrix. For 2-D retrievals, the row needs first to be reshaped into two dimensions, and then the FWHM method is used to calculate the resolution along each axis (Steck et al., 2005).

\section{Numerical experiments}

\subsection{Simulation setup}

In this section, we present the experimental results of tomographic temperature retrievals using simulated "target mode" measurements with $1 \%$ noise added. Synthetic measurements are generated by imprinting a GW structure onto a smooth model atmosphere, as described in Sect. 3.2. The temperature, atmospheric density, and concentrations of various constituents are perturbed by this simulated wave. As the amplitude is the most important feature of a GW with respect to energy, the assessment in the next step focuses on how well the wave amplitude can be reproduced from the retrieval results. The wave vector investigated in this case is assumed along the direction of line of sight, where the largest amplitude suppression is provided (Preusse et al., 2009b).
In our study, the spacing of the atmospheric grids is very important for both the forward and the retrieval model. To reduce the impact of the discretization on the synthetic measurements, the atmospheric grid in the forward model should be finely sampled. The atmospheric grid used in the forward model has a vertical spacing of $250 \mathrm{~m}$ and horizontal spacing of $5 \mathrm{~km}$. In the inverse procedure, the sampling can be coarser: $500 \mathrm{~m}$ vertical spacing and $12.5 \mathrm{~km}$ horizontal spacing in our case.

\subsection{Example of a GW parameter retrieval}

Figure 6 illustrates the performance of the tomographic retrieval approach for an atmosphere disturbed by a $\mathrm{GW}$ with a horizontal and vertical wavelength of 300 and $15 \mathrm{~km}$, respectively. The satellite platform is simulated in an approximately $600 \mathrm{~km}$ Sun-synchronous orbit with an inclination angle of $98^{\circ}$. The instrument will employ a 2-D detector array consisting of about $40 \times 400$ super pixels. It measures in the spectral regions from 13082 to $13103 \mathrm{~cm}^{-1}$ within the altitude range from $\approx 60$ to $120 \mathrm{~km}$ in limb imaging measurements. The atmospheric condition as well as the sampling patterns are the same as in the previous sections. The integration time is assumed to be $10 \mathrm{~s}$ for limb measurements and $15 \mathrm{~s}$ for sublimb measurements. The a priori data used in this case study are depicted in Fig. 6a. The simulated GW has an amplitude of $5 \mathrm{~K}$ (Fig. 6b). The retrieved temperature perturbation is shown in Fig. 6c. We can clearly see that the wave structure is well reproduced between 87 and $104 \mathrm{~km}$ height. The horizontal coverage of such a group of "target mode" measurements is around $800 \mathrm{~km}$. Figure $6 \mathrm{~d}$ depicts the difference between simulated wave and retrieved wave, with an average error of about $0.5 \mathrm{~K}$.

The spatial resolution of the retrieved data is usually described by the rows of the averaging kernel matrix, A. The deviation of $\mathbf{A}$ from the identity matrix gives insight into the smoothing introduced by the regularization. For example, if two adjacent grid points share one piece of information, the corresponding information content would be 0.5 . By reordering a single row of $\mathbf{A}$ according to their vertical and horizontal coordinates, the influence of each point on the retrieval result can be revealed. Figure 7 shows the 2-D averaging kernel matrix of two selected data points, which are marked as black dots in Fig. 6c.

Figure $7 \mathrm{a}$ shows the averaging kernel matrix for a point positioned at $2100 \mathrm{~km}$ along track and $95 \mathrm{~km}$ altitude. It indicates that the measurement contribution is sharply centred around this point. A minor part of the information comes from other altitudes on parabola shaped tracks, which correspond to the LOSs of observations, whose tangent altitude is below $95 \mathrm{~km}$ or which are sub-limb observations. For Fig. 7b, the data point is placed at $2100 \mathrm{~km}$ along track and $99 \mathrm{~km}$ altitude. In contrast to (a), this data point is not right on the tangent altitude of an observation, but in between two observations. Because this point is not placed exactly at one of the 
(a)

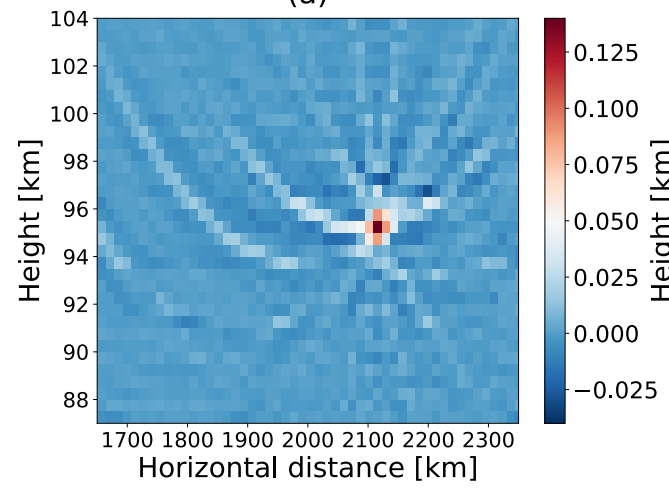

(b)

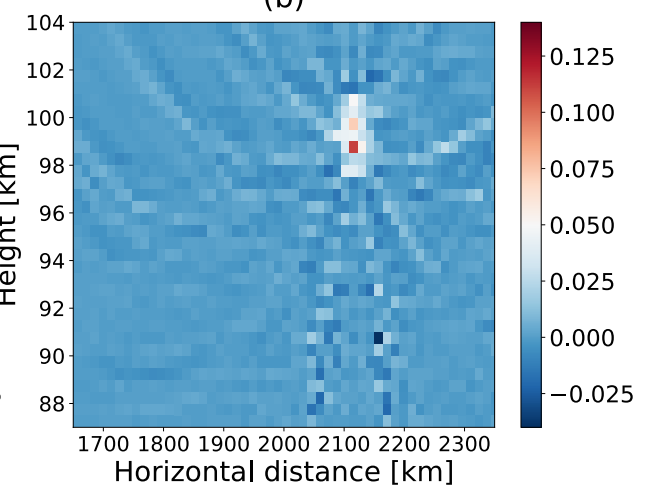

Figure 7. Averaging kernel matrix for two different retrieval points, allocated at the geographical position of highest values (red colour). Panel (a) shows a point coinciding with the observational grid, while panel (b) shows a point whose vertical position is in between two tangent altitudes of the limb observations; for details see text.

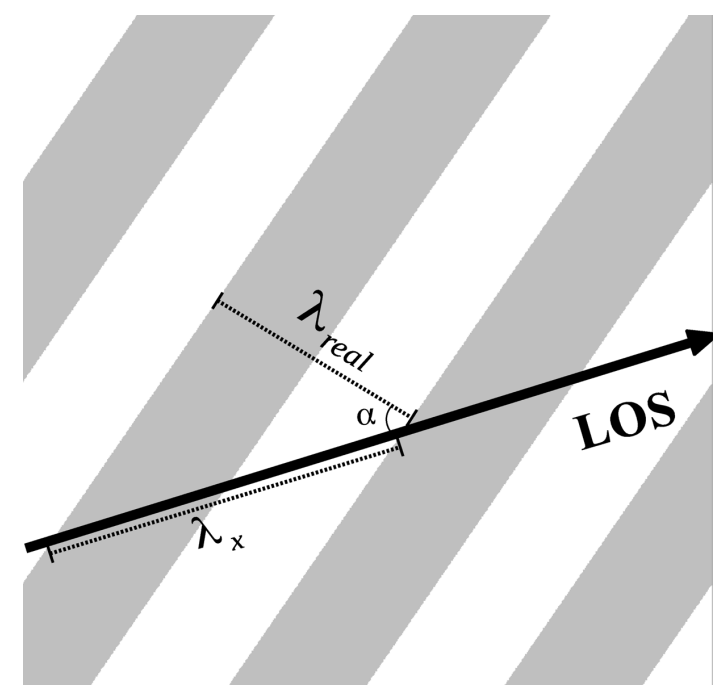

Figure 8. Viewing geometry between the satellite line of sight (LOS) and the horizontal wave vector. The wave fronts are represented by the grey shading. The observed horizontal wavelength $\lambda_{x}$ and the real horizontal wavelength $\lambda_{\text {real }}$ are related by an angle $\alpha$ : $\lambda_{\text {real }}=\lambda_{x} \cos \alpha$.

tangent altitudes, main contributions to the retrieved value come from measurements of adjacent grid points. According to Fig. $7 \mathrm{a}$, a vertical resolution of $1.3 \mathrm{~km}$ and horizontal resolution of $35 \mathrm{~km}$ can be achieved.

\subsection{Sensitivity study}

The quality of a GW amplitude-, wavelength- and phase- retrieval depends on the wavelength of the wave. The long LOS of limb observations is ideal for the reconstruction of long horizontal wavelengths, whereas GWs with short horizontal wavelengths are likely underestimated or cannot be measured at all. A measure to assess the sensitivity of an observation system to retrieve GW parameters is the so-called GW sensitivity function (Preusse et al., 2002). It defines how a wave perturbation of a given horizontal and vertical wavelength is reproduced by a retrieval. One option to determine this GW sensitivity function is to perform the retrieval for the wavelength of interest. However, for a tomographic retrieval this is computationally very expensive. Alternatively, we can derive the GW sensitivity function more efficiently by using the averaging kernel matrix method (Ungermann et al., 2010a). The basic idea of this method is to assume that the forward model can be approximated linearly for a small perturbation as induced by a GW. In this case, the averaging kernel matrix A would be identical for the unperturbed atmosphere and the perturbed atmosphere. If the unperturbed atmosphere $\boldsymbol{x}_{b}$ is assumed to be the same as the a priori data $\boldsymbol{x}_{a}$, the retrieval result $\boldsymbol{x}_{f}$ and the a priori vector $\boldsymbol{x}_{a}$ are related as follows:

$\boldsymbol{x}_{f}-\boldsymbol{x}_{a}=\left(\mathbf{A}\left(\boldsymbol{x}_{a}+\boldsymbol{x}_{\delta}\right)+(\mathbf{I}-\mathbf{A}) \boldsymbol{x}_{a}\right)-\boldsymbol{x}_{a}=\mathbf{A} \boldsymbol{x}_{\delta}$,

with $\mathbf{I}$ being the identity matrix and $\boldsymbol{x}_{\delta}$ being the modulated wave structure. Following this equation, the averaging kernel matrix A maps the true wave perturbations $\boldsymbol{x}_{\delta}$ onto the retrieved wave structure $\boldsymbol{x}_{f}-\boldsymbol{x}_{a}$. The ratio between $\boldsymbol{x}_{f}-\boldsymbol{x}_{a}$ and $\boldsymbol{x}_{\delta}$ quantifies the sensitivity to reconstruct the GW.

For this "target mode", the observed horizontal wavelength is the wavelength projected along the LOS. In general, there is an angle $\alpha$ between the LOS and the horizontal wave vector. Therefore, the observed horizontal wavelength $\lambda_{x}$ is a factor of $1 /(\cos \alpha)$ larger than the real horizontal wavelength $\lambda_{\text {real }}$, as illustrated in Fig. 8. In this sensitivity study, the horizontal wavelength discussed is the observed horizontal wavelength $\lambda_{x}$.

Figure 9 shows the results of sensitivity study adopting this approach for horizontal and vertical wavelengths of 0 400 and $0-60 \mathrm{~km}$, respectively. To illustrate the advancement of the combination of limb and sub-limb observations for the reconstruction of GW amplitudes, the sensitivity function for a pure limb measurement was also calculated, as shown in 
(a) Limb sounding

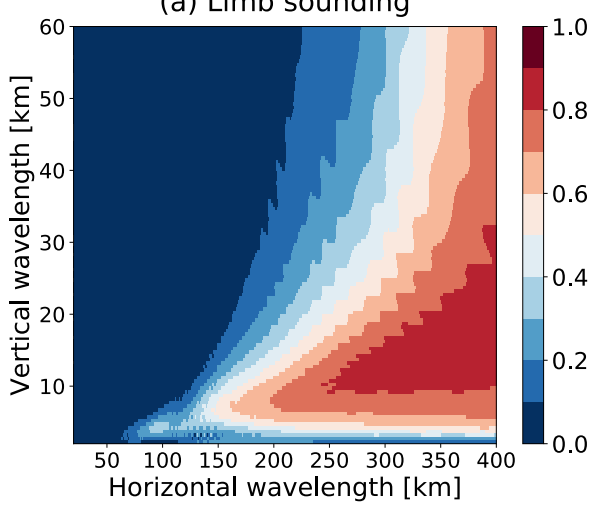

(b) "Target mode"

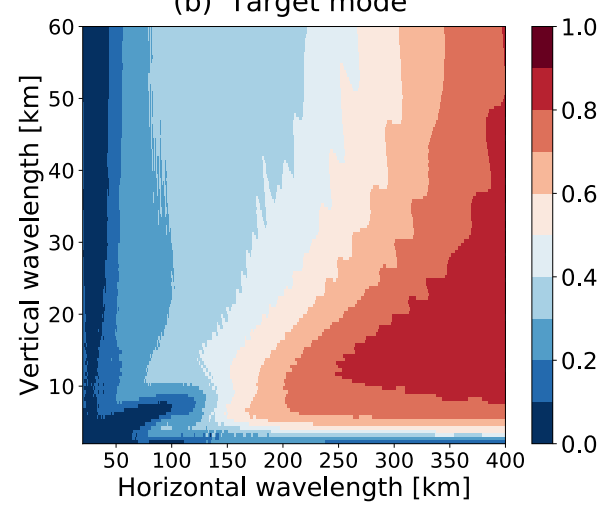

Figure 9. GW sensitivity function. The ratio of retrieved wave amplitude to true wave amplitude as function of horizontal and vertical wavelength is shown. Panel (a) is the sensitivity function for pure limb measurements, while panel (b) is for "target mode" measurements as specified in Sect. 5.

(a) Limb sounding

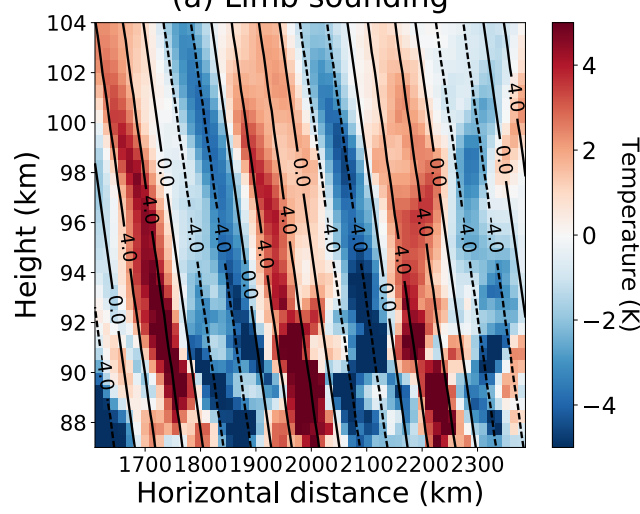

(b) "Target mode"

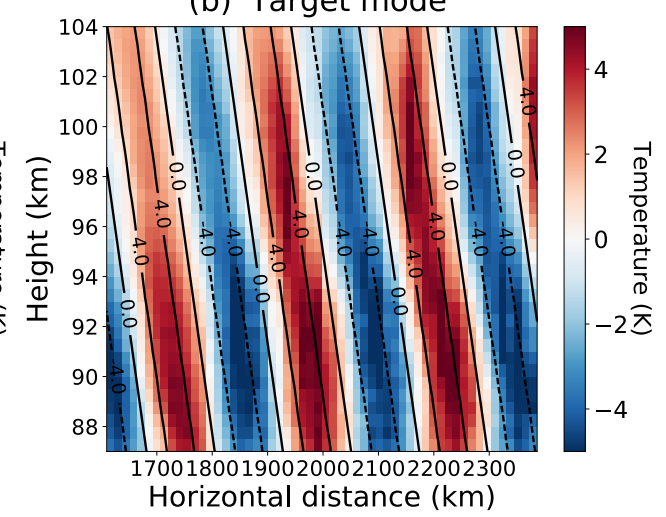

Figure 10. Pure limb sounding (a) and "target mode" (b) retrieval results comparison. The GW has a wavelength of $250 \mathrm{~km}$ in the horizontal and $40 \mathrm{~km}$ in the vertical. The climatological background profile was subtracted from the retrieval result. The black lines indicate the true modulated wave structure.

Fig. 9a. The retrieval setup is the same for both cases, but the additional sub-limb measurements were removed from Fig. $9 \mathrm{~b}$ to get the pure limb simulation in Fig. 9a.

For pure limb sounding, GWs with vertical and horizontal wavelengths down to 7 and $150 \mathrm{~km}$, respectively, can be observed. The sensitivity to detect short horizontal wavelengths decreases for larger vertical wavelengths, e.g. $250 \mathrm{~km}$ at $20 \mathrm{~km}$ vertical wavelength, or $325 \mathrm{~km}$ at $60 \mathrm{~km}$ vertical wavelength, respectively. In Fig. 9b, "target mode" tomography has been performed which considers sub-limb measurements with $15 \mathrm{~s}$ integration time as well. The GW sensitivity function does not change much for short vertical wavelength compared to pure limb measurements, but GWs with large vertical wavelength and short horizontal wavelength become much more visible. For GWs with vertical wavelengths above $15 \mathrm{~km}$, the increase in horizontal wavelength sensitivity is typically $50-100 \mathrm{~km}$.

Another advancement of the "target mode" is the reduced altitude dependence of the observational filter, as is illus- trated in Fig. 10 for a $\mathrm{GW}$ with $250 \mathrm{~km}$ horizontal wavelength and $40 \mathrm{~km}$ vertical wavelength. Comparing the retrieved wave structure with the true structure (depicted as the black contour plot), we find that the "target mode" tomography can reconstruct the wave structure more clearly than the limb mode tomography.

\section{Conclusions}

In recent years, tomographic retrieval approaches have been proposed to reconstruct 2-D GW structures. However, the spatial resolution of GWs retrieved from this observation mode is limited by the poor horizontal resolution along the LOS of these instruments.

In this paper, a novel "target mode" observation combining limb and sub-limb measurements for retrieval of GW parameters in the mesopause region is presented. A tailored 
retrieval scheme for this observational mode has been presented and its performance has been assessed.

We employed this new approach to simulated measurements of an instrument measuring the $\mathrm{O}_{2}$ A-band nightglow emissions to demonstrate its advantages in resolving 2-D atmospheric structures. The retrieval results show that a combination of limb and sub-limb measurements increases the sensitivity to detect short horizontal wavelengths by $50-100 \mathrm{~km}$ compared to pure limb sounding. GWs with vertical and horizontal wavelength down to 7 and $150 \mathrm{~km}$ can be resolved. It is shown that its capability of detecting short horizontal wavelength is dependent on the vertical wavelength of GWs, e.g. $200 \mathrm{~km}$ at $20 \mathrm{~km}$ vertical wavelength and $260 \mathrm{~km}$ at $60 \mathrm{~km}$ vertical wavelength.

Data availability. All data used in this work are available from the authors upon request (r.song@fz-juelich.de and m.kaufmann@fzjuelich.de)

Competing interests. The authors declare that they have no conflict of interest.

Acknowledgements. This work was supported in part by the National Natural Science Foundation of China under grant 41590852 and the China Scholarship Council (201404910513).

The article processing charges for this open-access

publication were covered by a Research

Centre of the Helmholtz Association.

Edited by: Markus Rapp

Reviewed by: two anonymous referees

\section{References}

Alexander, M. J. and Barnet, C.: Using Satellite Observations to Constrain Parameterizations of Gravity Wave Effects for Global Models, J. Atmos. Sci., 64, 1652-1665, https://doi.org/10.1175/JAS3897.1, 2007.

Alexander, M. J., Gille, J., Cavanaugh, C., Coffey, M., Craig, C., Eden, T., Francis, G., Halvorson, C., Hannigan, J., Khosravi, R., Kinnison, D., Lee, H., Massie, S., Nardi, B., Barnett, J., Hepplewhite, C., Lambert, A., and Dean, V.: Global estimates of gravity wave momentum flux from High Resolution Dynamics Limb Sounder observations, J. Geophys. Res.-Atmos., 113, d15S18, https://doi.org/10.1029/2007JD008807, 2008.

Buehler, S., Eriksson, P., Kuhn, T., von Engeln, A., and Verdes, C.: ARTS, the atmospheric radiative transfer simulator, J. Quant. Spectrosc. Ra., 91, 65-93, https://doi.org/10.1016/j.jqsrt.2004.05.051, 2005.

Burrage, M. D., Arvin, N., Skinner, W. R., and Hays, P. B.: Observations of the $\mathrm{O}_{2}$ atmospheric band nightglow by the high resolution Doppler imager, J. Geophys. Res., 99, 15017, https://doi.org/10.1029/94JA00791, 1994.

Campbell, I. and Gray, C.: Rate constants for $\mathrm{O}\left({ }^{3} P\right)$ recombination and association with $\mathrm{N}\left({ }^{4} S\right)$, Chem. Phys. Lett., 18, 607-609, https://doi.org/10.1016/0009-2614(73)80479-8, 1973.

Carlotti, M., Dinelli, B. M., Raspollini, P., and Ridolfi, M.: Geo-fit approach to the analysis of limb-scanning satellite measurements, Appl. Optics, 40, 1872-1885, https://doi.org/10.1364/ORS.2001.OWC5, 2001.

Ceccherini, S. and Ridolfi, M.: Technical Note: Variance-covariance matrix and averaging kernels for the Levenberg-Marquardt solution of the retrieval of atmospheric vertical profiles, Atmos. Chem. Phys., 10, 3131-3139, https://doi.org/10.5194/acp-103131-2010, 2010.

Eckermann, S. D. and Preusse, P.: Global Measurements of Stratospheric Mountain Waves from Space, Science, 286, 1534-1537, https://doi.org/10.1126/science.286.5444.1534, 1999.

Eriksson, P., Buehler, S., Davis, C., Emde, C., and Lemke, O.: ARTS, the atmospheric radiative transfer simulator, version 2, J. Quant. Spectrosc. Ra., 112, 1551-1558, https://doi.org/10.1016/j.jqsrt.2011.03.001, 2011.

Ern, M., Preusse, P., Alexander, M. J., and Warner, C. D.: Absolute values of gravity wave momentum flux derived from satellite data, J. Geophys. Res.-Atmos., 109, d20103, https://doi.org/10.1029/2004JD004752, 2004.

Ern, M., Lehmann, C., Kaufmann, M., and Riese, M.: Spectral wave analysis at the mesopause from SCIAMACHY airglow data compared to SABER temperature spectra, Ann. Geophys., 27, 407416, https://doi.org/10.5194/angeo-27-407-2009, 2009.

Ern, M., Preusse, P., Gille, J. C., Hepplewhite, C. L., Mlynczak, M. G., Russell, J. M., and Riese, M.: Implications for atmospheric dynamics derived from global observations of gravity wave momentum flux in stratosphere and mesosphere, J. Geophys. Res.-Atmos., 116, d19107, https://doi.org/10.1029/2011JD015821, 2011.

Ern, M., Hoffmann, L., and Preusse, P.: Directional gravity wave momentum fluxes in the stratosphere derived from highresolution AIRS temperature data, Geophys. Res. Lett., 44, 475485, https://doi.org/10.1002/2016GL072007, 2016GL072007, 2017.

Fetzer, E. J. and Gille, J. C.: Gravity Wave Variance in LIMS Temperatures. Part I: Variability and Comparison with Background Winds, J. Atmos. Sci., 51, 2461-2483, https://doi.org/10.1175/15200469(1994)051<2461:GWVILT>2.0.CO;2, 1994.

Fetzer, E. J. and Gille, J. C.: Gravity Wave Variance in LIMS Temperatures. Part II: Comparison with the Zonal-Mean Momentum Balance, J. Atmos. Sci., 53, 398-410, https://doi.org/10.1175/15200469(1996)053<0398:GWVILT>2.0.CO;2, 1996.

Forbes, J. M., Russell, J., Miyahara, S., Zhang, X., Palo, S., Mlynczak, M., Mertens, C. J., and Hagan, M. E.: Tropospherethermosphere tidal coupling as measured by the SABER instrument on TIMED during July-September 2002, J. Geophys. Res.Space, 111, a10S06, https://doi.org/10.1029/2005JA011492, 2006.

Fritts, D. C. and Alexander, M. J.: Gravity wave dynamics and effects in the middle atmosphere, Rev. Geophys., 41, 1003, https://doi.org/10.1029/2001RG000106, 2003. 
Fritts, D. C., Pautet, P.-D., Bossert, K., Taylor, M. J., Williams, B. P., Iimura, H., Yuan, T., Mitchell, N. J., and Stober, G.: Quantifying gravity wave momentum fluxes with Mesosphere Temperature Mappers and correlative instrumentation, J. Geophys. Res.-Atmos., 119, 2014JD022150, 13583-13603, https://doi.org/10.1002/2014JD022150, 2014.

Garcia, R. R. and Solomon, S.: The effect of breaking gravity waves on the dynamics and chemical composition of the mesosphere and lower thermosphere, J. Geophys. Res., 90, 3850, https://doi.org/10.1029/JD090iD02p03850, 1985.

Gong, J., Wu, D. L., and Eckermann, S. D.: Gravity wave variances and propagation derived from AIRS radiances, Atmos. Chem. Phys., 12, 1701-1720, https://doi.org/10.5194/acp-121701-2012, 2012.

Greer, R., Llewellyn, E., Solheim, B., and Witt, G.: The excitation of $\mathrm{O}_{2}\left(b_{1} \Sigma_{g}^{+}\right)$in the nightglow, Planet. Space Sci., 29, 383-389, https://doi.org/10.1016/0032-0633(81)90081-7, 1981.

Hoffmann, L. and Alexander, M. J.: Retrieval of stratospheric temperatures from Atmospheric Infrared Sounder radiance measurements for gravity wave studies, J. Geophys. Res.-Atmos., 114, d07105, https://doi.org/10.1029/2008JD011241, 2009.

Hoffmann, L., Grimsdell, A. W., and Alexander, M. J.: Stratospheric gravity waves at Southern Hemisphere orographic hotspots: 2003-2014 AIRS/Aqua observations, Atmos. Chem. Phys., 16, 9381-9397, https://doi.org/10.5194/acp-16-9381-2016, 2016.

Holton, J. R.: The Role of Gravity Wave Induced Drag and Diffusion in the Momentum Budget of the Mesosphere, J. Atmos. Sci., 39, 791-799, https://doi.org/10.1175/15200469(1982)039<0791:TROGWI>2.0.CO;2, 1982.

Kaufmann, M., Blank, J., Guggenmoser, T., Ungermann, J., Engel, A., Ern, M., Friedl-Vallon, F., Gerber, D., Grooß, J. U., Guenther, G., Höpfner, M., Kleinert, A., Kretschmer, E., Latzko, Th., Maucher, G., Neubert, T., Nordmeyer, H., Oelhaf, H., Olschewski, F., Orphal, J., Preusse, P., Schlager, H., Schneider, H., Schuettemeyer, D., Stroh, F., Suminska-Ebersoldt, O., Vogel, B., M. Volk, C., Woiwode, W., and Riese, M.: Retrieval of threedimensional small-scale structures in upper-tropospheric/lowerstratospheric composition as measured by GLORIA, Atmos. Meas. Tech., 8, 81-95, https://doi.org/10.5194/amt-8-81-2015, 2015.

Levenberg, K.: A method for the solution of certain non-linear problems in least squares, Q. Appl. Math., 2, 164-168, 1944.

Lindzen, R. S.: Turbulence and stress owing to gravity wave and tidal breakdown, J. Geophys. Res., 86, 9707, https://doi.org/10.1029/JC086iC10p09707, 1981.

Liu, A. Z. and Swenson, G. R.: A modeling study of $\mathrm{O}_{2}$ and $\mathrm{OH}$ airglow perturbations induced by atmospheric gravity waves, J. Geophys. Res., 108, 4151, https://doi.org/10.1029/2002JD002474, 2003.

Livesey, N. J. and Read, W. G.: Direct retrieval of line-of-sight atmospheric structure from limb sounding observations, Geophys. Res. Lett., 27, 891-894, https://doi.org/10.1029/1999GL010964, 2000

Marquardt, D. W.: An Algorithm for Least-Squares Estimation of Nonlinear Parameters, J. Soc. Ind. Appl. Math., 11, 431-441, https://doi.org/10.1137/0111030, 1963.

McDade, I., Murtagh, D., Greer, R., Dickinson, P., Witt, G., Stegman, J., Llewellyn, E., Thomas, L., and Jenkins, D.: ETON 2: Quenching parameters for the proposed precursors of
$\mathrm{O}_{2}\left(b_{1} \Sigma_{g}^{+}\right)$and $\mathrm{O}\left({ }^{1} \mathrm{~S}\right)$ in the terrestrial nightglow, Planet. Space Sci., 34, 789-800, https://doi.org/10.1016/0032-0633(86)900759, 1986.

Preusse, P., Dörnbrack, A., Eckermann, S. D., Riese, M., Schaeler, B., Bacmeister, J. T., Broutman, D., and Grossmann, K. U.: Space-based measurements of stratospheric mountain waves by CRISTA 1 . Sensitivity, analysis method, and a case study, J. Geophys. Res.-Atmos., 107, 8178, https://doi.org/10.1029/2001JD000699, 2002.

Preusse, P., Eckermann, S. D., Ern, M., Oberheide, J., Picard, R. H., Roble, R. G., Riese, M., Russell, J. M., and Mlynczak, M. G.: Global ray tracing simulations of the SABER gravity wave climatology, J. Geophys. Res.-Atmos., 114, d08126, https://doi.org/10.1029/2008JD011214, 2009a.

Preusse, P., Schroeder, S., Hoffmann, L., Ern, M., Friedl-Vallon, F., Ungermann, J., Oelhaf, H., Fischer, H., and Riese, M. New perspectives on gravity wave remote sensing by spaceborne infrared limb imaging, Atmos. Meas. Tech., 2, 299-311, https://doi.org/10.5194/amt-2-299-2009, 2009b.

Riese, M., Oelhaf, H., Preusse, P., Blank, J., Ern, M., Friedl-Vallon, F., Fischer, H., Guggenmoser, T., Höpfner, M., Hoor, P., Kaufmann, M., Orphal, J., Plöger, F., Spang, R., Suminska-Ebersoldt, O., Ungermann, J., Vogel, B., and Woiwode, W.: Gimballed Limb Observer for Radiance Imaging of the Atmosphere (GLORIA) scientific objectives, Atmos. Meas. Tech., 7, 1915-1928, https://doi.org/10.5194/amt-7-1915-2014, 2014.

Rodgers, C. D.: Inverse methods for atmospheric sounding: theory and practice, no. 2 in Series on atmospheric oceanic and planetary physics, World Scientific, Singapore, reprinted Edn., 2004.

Schmidt, H., Brasseur, G. P., Charron, M., Manzini, E., Giorgetta, M. A., Diehl, T., Fomichev, V. I., Kinnison, D., Marsh, D., and Walters, S.: The HAMMONIA Chemistry Climate Model: Sensitivity of the Mesopause Region to the 11-Year Solar Cycle and $\mathrm{CO}_{2}$ Doubling, J. Climate, 19, 3903-3931, https://doi.org/10.1175/JCLI3829.1, 2006.

Sheese, P.: Mesospheric ozone densities retrieved from OSIRIS observations of the $\mathrm{O}_{2}$ A-band dayglow, PhD thesis, Library and Archives Canada - Bibliothèque et Archives Canada, Ottawa, 2011.

Steck, T., Höpfner, M., von Clarmann, T., and Grabowski, U.: Tomographic retrieval of atmospheric parameters from infrared limb emission observations, Appl. Optics, 44, 3291, https://doi.org/10.1364/AO.44.003291, 2005.

Tikhonov, A. N. and Arsenin, V. I.: Solutions of ill-posed problems, Scripta series in mathematics, Winston; distributed solely by Halsted Press, Washington: New York, 1977.

Tsuda, T., Nishida, M., Rocken, C., and Ware, R. H.: A Global Morphology of Gravity Wave Activity in the Stratosphere Revealed by the GPS Occultation Data (GPS/MET), J. Geophys. Res.-Atmos., 105, 7257-7273, https://doi.org/10.1029/1999JD901005, 2000.

Ungermann, J., Hoffmann, L., Preusse, P., Kaufmann, M., and Riese, M.: Tomographic retrieval approach for mesoscale gravity wave observations by the PREMIER Infrared Limb-Sounder, Atmos. Meas. Tech., 3, 339-354, https://doi.org/10.5194/amt-3339-2010, 2010a.

Ungermann, J., Kaufmann, M., Hoffmann, L., Preusse, P., Oelhaf, H., Friedl-Vallon, F., and Riese, M.: Towards a 3-D tomographic retrieval for the air-borne limb-imager GLORIA, Atmos. Meas. 
Tech., 3, 1647-1665, https://doi.org/10.5194/amt-3-1647-2010, 2010b.

Ungermann, J., Blank, J., Lotz, J., Leppkes, K., Hoffmann, L., Guggenmoser, T., Kaufmann, M., Preusse, P., Naumann, U., and Riese, M.: A 3-D tomographic retrieval approach with advection compensation for the air-borne limb-imager GLORIA, Atmos. Meas. Tech., 4, 2509-2529, https://doi.org/10.5194/amt-4-25092011, 2011.

Vallance Jones, A.: Aurora Geophysics and Astrophysics Monographs, D. Reidel, Hingham, Mass, 1974.

Vargas, F., Swenson, G., Liu, A., and Gobbi, D.: $\mathrm{O}\left({ }^{1} \mathrm{~S}\right), \mathrm{OH}$, and $\mathrm{O}_{2}$ (b) airglow layer perturbations due to AGWs and their implied effects on the atmosphere, J. Geophys. Res., 112, D14102, https://doi.org/10.1029/2006JD007642, 2007.

Vincent, R. A.: The dynamics of the mesosphere and lower thermosphere: a brief review, Prog. Earth Planet. Sci., 2, https://doi.org/10.1186/s40645-015-0035-8, 2015. von Clarmann, T., De Clercq, C., Ridolfi, M., Höpfner, M., and Lambert, J.-C.: The horizontal resolution of MIPAS, Atmos. Meas. Tech., 2, 47-54, https://doi.org/10.5194/amt-2-47-2009, 2009.

Ward, W. E.: A simple model of diurnal variations in the mesospheric oxygen nightglow, Geophys. Res. Lett., 26, 3565-3568, https://doi.org/10.1029/1999GL003661, 1999.

Wright, C. J., Osprey, S. M., Barnett, J. J., Gray, L. J., and Gille, J. C.: High Resolution Dynamics Limb Sounder measurements of gravity wave activity in the 2006 Arctic stratosphere, J. Geophys. Res.-Atmos., 115, d02105, https://doi.org/10.1029/2009JD011858, 2010.

$\mathrm{Wu}$, D. L.: Mesoscale gravity wave variances from AMSU-A radiances, Geophys. Res. Lett., 31, 112114, https://doi.org/10.1029/2004GL019562, 2004. 